\title{
Evaluating Mixed-Precision Arithmetic for 3D Generative Adversarial Networks to Simulate High Energy Physics Detectors
}

\author{
John Osorio Ríos*†, Adrià Armejach*†, Gulrukh Khattak ${ }^{\ddagger}$, Eric Petit ${ }^{\S}$, Sofia Vallecorsa ${ }^{\ddagger}$ and Marc Casas*† \\ *Barcelona Supercomputing Center (BSC), Barcelona, Spain \\ ${ }^{\dagger}$ Universitat Politècnica de Catalunya (UPC), Barcelona, Spain \\ ${ }^{\ddagger}$ CERN, Geneva, Switzerland \\ $\S$ Intel, Paris, France \\ Email: john.osorio@bsc.es
}

\begin{abstract}
Several hardware companies are proposing native Brain Float 16-bit (BF16) support for neural network training. The usage of Mixed Precision (MP) arithmetic with floating-point 32-bit (FP32) and 16-bit half-precision aims at improving memory and floating-point operations throughput, allowing faster training of bigger models. This paper proposes a binary analysis tool enabling the emulation of lower precision numerical formats in Neural Network implementation without the need for hardware support. This tool is used to analyze BF16 usage in the training phase of a 3D Generative Adversarial Network (3DGAN) simulating High Energy Physics detectors. The binary tool allows us to confirm that BF16 can provide results with similar accuracy as the full-precision 3DGAN version and the costly reference numerical simulation using double precision arithmetic.
\end{abstract}

Index Terms-Reduced Precision, Brain Float 16 (BF16), Mixed Precision (MP), 3DGAN, Binary Analysis Tool, High Energy Physics, Generative Adversarial Networks.

\section{INTRODUCTION}

Following the progress made in image classification tasks [1], [2] by Khrizhevsky et al. [3], Szegedy et al. [4] and He et al. [5], Deep Neural Networks (DNNs) [6] have been successfully applied to domains like biology [7], economy [8], chemistry [9], or sports management [10]. Many refined techniques have emerged to tackle these new problems beyond image classification. Among them, the Generative Adversarial Networks (GAN) proposed by Goodfellow et al. [11]. CERN has recently proposed to drive High Energy Physics (HEP) simulations using GANs [12]-[16].

Models based on DNNs must be trained on large sets of data to reach acceptable accuracy levels. Training incurs very large computational and memory training costs. Several novel techniques are aimed at decreasing the training costs by avoiding the use of floating-point 32-bit (FP32) numerical datatype, and replacing it with non-standard low precision data representations formats [17]-[19]. Recent hardware products support 16bit half-precision to accelerate training. Micikevicius et al. [20] and Kalamkar et al. [21], show how to train DNNs using Mixed Precision (MP) approaches. A combination of FP32 and half-precision representations using either the floating-

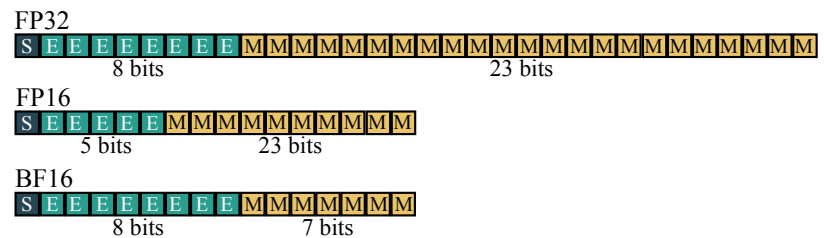

Fig. 1: Floating-point Formats. S sign bit, E exponent bits, M mantissa bits.

point 16 (FP16) or the Brain floating-point (BF16) datatypes, which we display in Figure 1, reaches the same accuracy levels as FP32 arithmetic for many DNN applications. A strong emphasis is given to Fused Multiply-Add (FMA) instructions computing $A \cdot B+C$, which represent the largest part of the computational time. The MP approach for FMAs uses FP32 precision to accumulate the contributions of $A \cdot B$ and $C$, while the multiplication uses half-precision inputs, as seen in Figure 2 .

In this paper, we adapt and demonstrate the capacity of MP arithmetic to train GANs driving HEP simulations. In particular, we focus in MP FMAs combining BF16 and FP32, which Figure $2 \mathrm{~b}$ represents.

We leverage a binary analysis tool based on PIN [22] to emulate lower precision numerical formats without the need of native hardware support. The binary analysis tool intercepts FMA instructions and modifies its operands at the register level to emulate low-precision arithmetic. In particular, we use the Rounding to Nearest Even (RNE) to convert FP32 values to the BF16 format. Once the conversion is done, our binary analysis tool gives the control back to the training process, which implies that the FMA instruction is natively executed with some of its operands truncated to 16-bits representation. Our tool can be applied to either the PyTorch [23], the Caffe [24], or the Tensorflow [25] frameworks.

Our evaluation shows that $98 \%$ of floating-point instructions are FMAs, and that we just need to compute in full singleprecision (FP32) the $0.001 \%$ of them, as Section $\mathrm{V}$ indicates.

(c) 2020 IEEE. Personal use of this material is permitted. Permission from IEEE must be obtained for all other uses, in any current or future media, including reprinting/republishing this material for advertising or promotional purposes,creating new collective works, for resale or redistribution to servers or lists, or reuse of any copyrighted component of this work in other works. 
In addition, we empirically validate that MP FMAs using BF16 are able to train GAN models with the similar accuracy as full FP32, and a real physical Monte Carlo reference simulation. Section VI contains this evaluation. To the best of our knowledge, this is the first proposal that trains GAN models using MP and applies them to HEP simulation problems.

\section{MiXed PRECision Training}

BF16 is a numerical datatype created by Google used in their Google Brain group [26]. It was initially created to reduce the amount of data shared by different computing nodes during distributed DNN training, and used specifically on their Tensor Processing Units (TPU). However, since BF16 has the same dynamic range as FP32, as both have the same number of exponent bits (see Figure 1), the conversion between these two formats is very simple and it shows great robustness while training DNNs. On the other hand, the floating-point 16 (FP16) data type requires scaling factors to perform the conversion from/to FP32. For these reasons, BF16 has become a reference datatype with hardware support announced for multiple upcoming computer architectures from vendors like Nvidia [27], Intel [28], and Arm [29].

However, training with BF16 requires careful attention to arithmetic sensitive parts of the algorithm such as weights updates, batch normalization, and gradients summation. This leads to the usage of a MP approach, where a combination of FP32 and BF16 is used in different places and flavors. When employing MP, FMA instructions (MP FMA) combine halfprecision and FP32 input values and usually accumulate in FP32 as shown in Figure 2

Figure 2a details the type of FMA instruction employed in Nvidia's MP training [20]. An FMA instruction computes $D=A \cdot B+C$. Input parameters $A$ and $B$ are represented in FP16, the result is added to the third input $C$, typically a weight of the master copy represented in FP32. The final output $D$ is also represented in FP32. This approach requires additional steps to enforce that FP32 values that are converted into FP16 fall into the representative range of the latter by applying a scaling factor.

Figure 2b shows the FMA implementation used by Kalamkar et al. in [21], which is similar to the one previously described. The only difference is about the low precision datatype used to do the calculations which is BF16, simplifying the conversion from/to FP32. Since the BF16 data type is only available on the proprietary Google TPU, this study of MP employing BF16 was performed without hardware support, by modifying the evaluated neural networks to perform library calls to do the conversion from FP32 to BF16 in software, using truncation and RNE rounding. In this regard, Intel's next generation family of Intel Xeon processors, code named Cooper Lake, will incorporate BF16 hardware support to use MP approaches in the training processes of DNN [28].

Additionally, there is another possible FMA instruction implementation, as shown in Figure 2c where all inputs and outputs are represented using BF16. However, using this approach during training does not provide good converge properties for the most popular DNNs. This is caused by the absorption issue during the addition step in the FMA instruction. That is, when right-shifting the smaller of the two operands to align bits with the same exponents, the lowest bit cannot be represented in the rounded result. The smaller number can even be entirely absorbed due to the limited number of mantissa bits, this issue is known as swamping [19] or absorption in the computer arithmetic field, and leads to information loss when the magnitudes of the added operands have a difference $>2^{\text {mantissa+1 }}$ bits. In the case of BF16 with 7 mantissa bits, the swamping threshold is 256 .

Regardless of the MP FMA implementation used, some phases of the training process require FP32 precision to keep the same accuracy when training the DNN models. This is the case of the weight updates (WU), batch normalization $(\mathrm{BN})$, and soft-max layers.

In this paper we demonstrate the feasibility to apply MP training to GAN models for HEP simulations. The following section introduces a three-dimensional GAN model and outlines the computational challenges it presents, which can be mitigated by applying MP. Therefore, the use of MP can help advance this field by enabling larger input datasets or models with additional complexity that can be trained in a sensitive amount of time, with the objective to achieve better simulation results.

\section{The 3D Convolutional GAN}

Within the field of HEP, Monte Carlo simulation represents a heavy computational task, and generative models are being studied as possible alternatives [12]-[14]. 3DGAN [15] represents the first application of three-dimensional convolutional Generative Adversarial Networks to the simulation of high granularity electromagnetic calorimeters, detectors that can measure particles energy with a high level of precision. The aim of the study is to produce a network which can receive as input a particle type and energy, and which will produce an accurate simulation of the corresponding particle detector output. In particular, the detector geometry is chosen to be an example of future calorimeters, characterized by a regular grid of small sensitive cubic cells $\left(5.1 \mathrm{~mm}^{3}\right)$. Individual electrons, photons, charged pions, and neutral pions are shot into the calorimeter volume at various energies and represent a single event in the training data set. For each event we take a $25 \times 25 \times 25$ cell volume of the electromagnetic calorimeter (ECAL) and store them as a 3D array containing information about the energy deposited in each cell.

3DGAN implements an architecture that is similar to the Auxiliary Classifier Generative Adversarial Network [30] the generator and discriminator models consist of four $3 \mathrm{D}$ convolution layers and the discriminator performs an auxiliary task by estimating the particle energy. Details of the architecture can be found in Figure 3. Leaky ReLU activation functions are used for the discriminator network layers. A batch normalization layer is added after all activations except the first layer. The output of the final convolution layer is flattened and connected to a sigmoid neuron corresponding to 


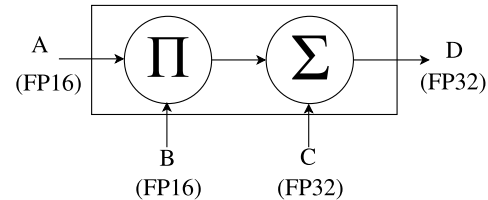

(a) MP FMA with FP16 and FP32 inputs

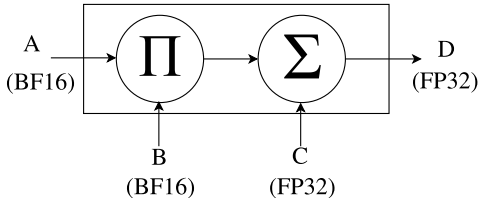

(b) MP FMA with BF16 and FP32 inputs

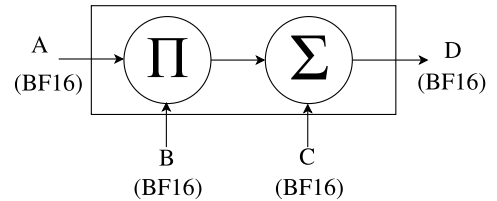

(c) BF16 FMA where all inputs are BF16

Fig. 2: Different alternatives when computing a Fused Multiply-Add (FMA) instruction.
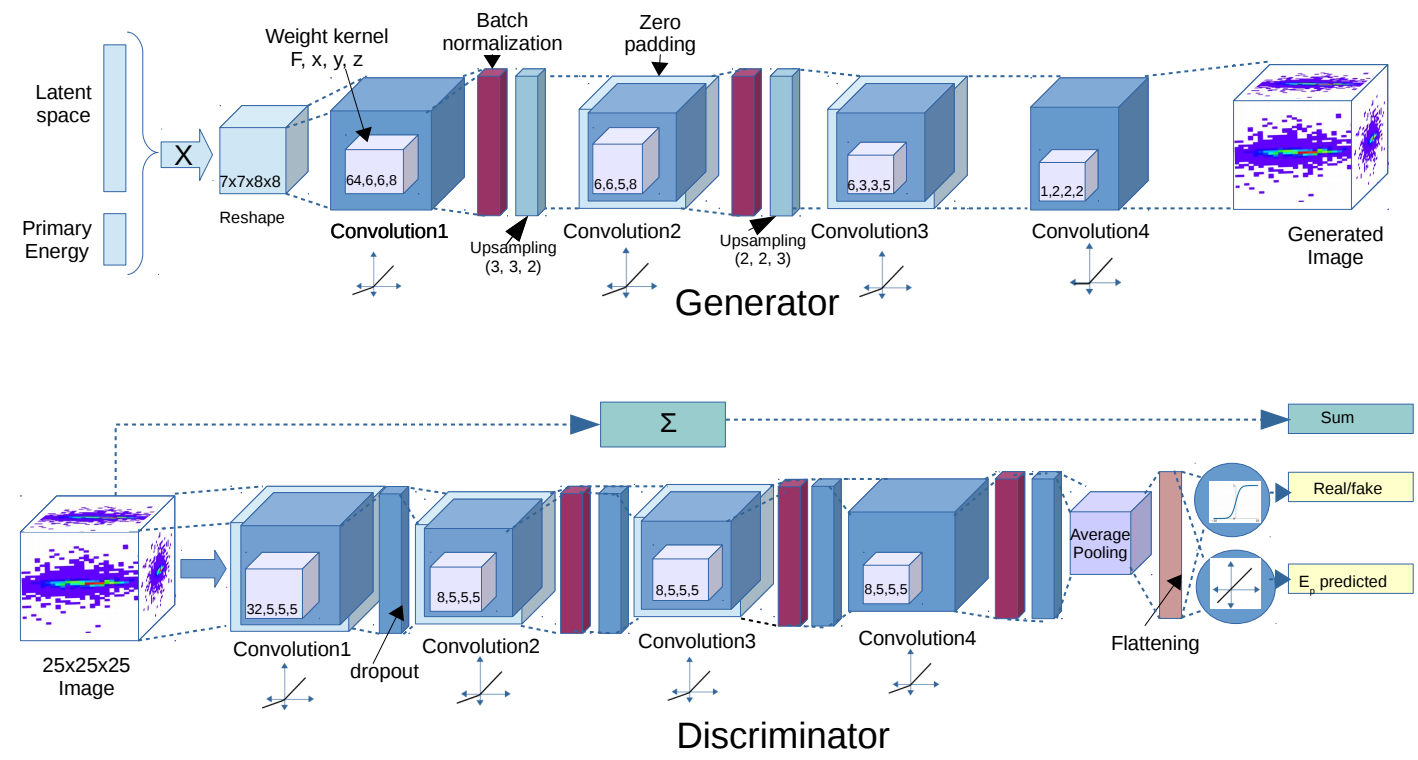

Fig. 3: 3DGAN model architecture.

real/fake output of GAN as well as a linear unit for energy regression. The generator has a latent vector of size 128 and a similar architecture with leaky ReLU (ReLU for the last layer) activation functions. Batch normalization layers are added after the first and second layers. The GAN cost function is modified to include an auxiliary energy regression task as well as checks on total energy deposited in order to constrain the distribution of individual cell energies.

GANs are a class of networks that are used to learn and reproduce the results of complex data distributions. As later explained in section $\mathrm{VI}$, the 3DGAN performance is compared to the classical Monte Carlo approach. Monte Carlo simulation requires a high level of accuracy, the more precise the simulated data set in terms of physics results, the smaller the uncertainty will be on the final physics measurement. In order to keep a similar level of accuracy both the 3DGAN training and inference process use FP32 by default. Unfortunately, as models in the HEP field become more complex, they also become more expensive in terms of memory capacity and memory bandwidth. 3DGAN represent a relatively lightweight prototype optimised to simulate a small portion of a HEP detector, in order to develop it into a production ready algorithm, capable of generating data for full scale detectors, memoryrelated challenges need to be addressed. A similar constraint prevents today the full optimisation of image reconstruction models, used to analyse the output of state-of-the-art neutrino detectors, such as Liquid Argon Time Projection Chambers (LAr TPC) [31]. TPC produce sets of images of such size (millions of pixels) that segmentation is today the only viable approach to their analysis. The possibility to reduce the memory footprint in such cases would certainly improve the final physics results. As discussed below, the MP approach helps improve this situation while maintaining the results accuracy at a reasonable level.

\section{MP EMULATION TOOL}

Without available hardware implementing the BF16 numerical format, several approaches have been used in the past to emulate the behaviour of reduced floating-point representations. Most notably, these efforts have focused on libraries that perform transformations like truncation and rounding [21], [32]. However, such an approach requires a significant effort to port every new target neural network to use these libraries, leading to a tedious process that needs to be repeated for each network.

To have a generic mechanism that can be seamlessly applied to any neural network and DNN framework, we have developed a binary level emulation tool. This tool enables 


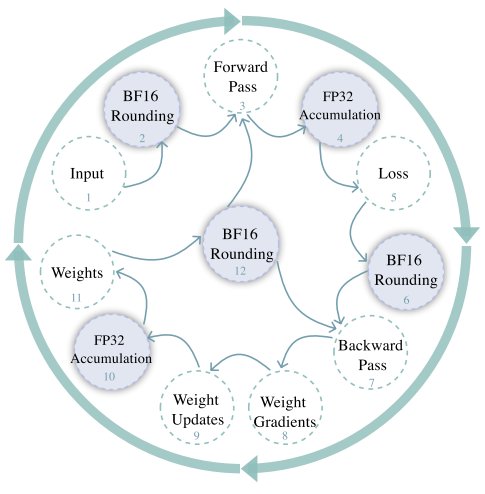

Fig. 4: Overview of the MP training process.

evaluating the impact that MP has on the accuracy of a target model. Our binary tool is based on Intel PIN 3.7 [22], a dynamic instrumentation framework for x86_64 architectures. The tool captures and instruments dynamic instructions, which enables modifying numerical operands to the targeted numerical data format. Our approach seamlessly works on complex frameworks like PyTorch, Tensorflow, or Caffe, with interpreted languages, and is able to instrument instructions triggered by dynamically linked libraries. One of the most important advantages of our tool is the capacity to adapt to new DNN frameworks without any or minimum effort to support them. Despite having no significant overhead in memory consumption, out tool induces a reasonable slowdown of 7 to $20 x$ over the original training time. This allows full training of full size data-sets with a conventional level of hardware resources.

Our binary analysis tool performs the following steps:

- Checks the current execution routine to determine if we are executing routines that belong to weight update or batch normalization layers. In that case, computation proceeds in FP32.

- The tool intercepts all floating-point instructions of the workload, including FMAs. For each FMA instruction, operands that need to be rounded to BF16, depending on the current routine, are rounded using RNE algorithm.

- The tool can dynamically change its operation mode via a simple inter-process communication method that can be invoked from the python high-level interface, this is useful to test some additional scenarios, or to avoid instrumenting all the preprocessing steps involved during the training process of DNN.

Figure 4 shows an overview of the steps needed to implement MP. As can bee seen in the figure, the input to the network is typically represented with FP32 values. Our binary analysis tool seamlessly converts the FMA inputs $A$ and $B$ to BF16 using RNE rounding on all forward pass computations. These are then accumulated in FP32, as defined in MP training (see Figure 2b). Similarly, in the backward step the FMA inputs $A$ and $B$ are again converted to BF16, and the final result in FP32 contains the weight gradients.

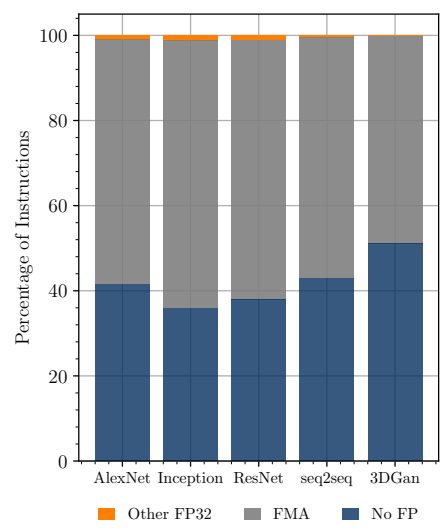

Fig. 5: Instruction Breakdown for multiple neural network models.

Finally, the weight gradients will be used to update the weights using FP32 arithmetic, which is crucial [19]-[21] to obtain the same accuracy as state-of-the-art FP32 training methods. These steps are repeated for each batch during the training process of the 3DGAN model. Note that the amount of computation (FMAs) done during the forward and backward passes is orders of magnitude larger than computation done in the layers that employ FP32 arithmetic. More details about this in Section V-B

\section{Evaluation Methodology}

\section{A. Evaluation platform and Training Parameters}

Our experiments are performed on Intel Xeon Platinum 8160 processors, which include the AVX512 ISA extension. To train and test the 3DGAN implementation we use Tensorflow 1.15 [25] and Keras 2 [33]. We use Intel MKLDNN [34] Deep Neural Network library 0.18.0 and the Intel MKL 2019.0.3 library [35] to run numerical kernels since both libraries are optimized to run on our testing infrastructure. The binary analysis tool uses PIN 3.7 and is compiled with GCC 8.0.

The 3DGAN network is trained for 60 epochs using the Adam optimiser and a batch size of 128 . The training dataset consists of 180,000 $25 \times 25 \times 25$ three-dimensional images generated using classical Monte Carlo, each representing a single electron of variable energy entering the detector volume.

\section{B. Use case characterization}

Figure 5 shows the percentage of instructions being processed by several DNN models. While training DNNs the compute units (GPU, CPU) perform, due to the nature of neural network architectures, a substantial amount of additions and multiplications. These in the end are calculated using specialized hardware instructions inside the computing units, called FMAs. As shown in Figure 5, FMA instructions represent a large portion of the overall instruction mix. The Other FP32 category are floating-point instructions that are not FMA, and No FP are integer, memory, and branch instructions.

In the figure, well known DNN models such as AlexNet [3], Inception [4], ResNet [5] and seq2seq [36], [37] are shown 


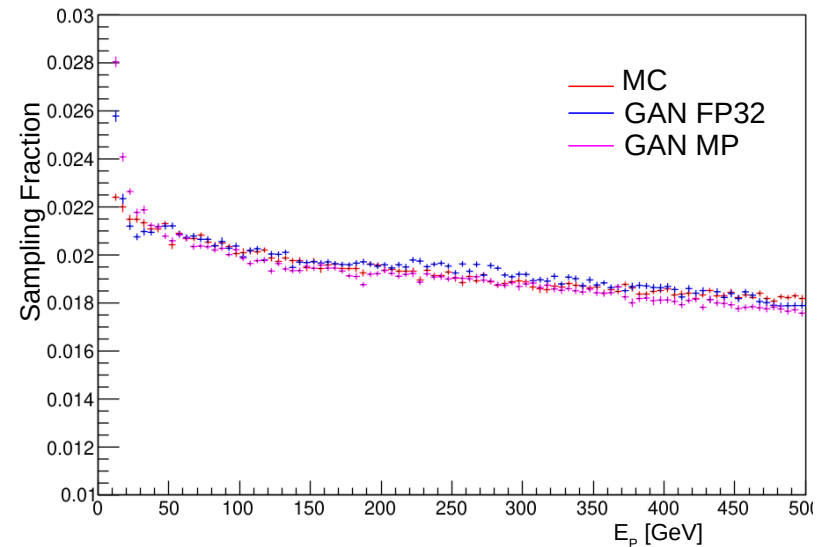

Fig. 6: Sampling Fraction (SF) vs. particle energy profile histograms for simulation using Monte Carlo (MC) (red), GAN FP32 (blue) and GAN MP (magenta).

to stress the importance FMA instructions have on any DNN training process. The percentage of FMA instructions for these models is $57.42 \%, 60.93 \%, 62.95 \%$, and $56.44 \%$, respectively. In 3DGAN, our use case, $374 * 10^{9}$ instructions are executed during one training batch, the percentage of FMAs is of $48.80 \%$, which is a significant portion, opening an opportunity to apply a technique such as MP to improve throughput and reduce bandwidth requirements. Note that, as mentioned before, certain layers or routines still need to be computed using FP32 (weight updates, batch normalization and softmax); however, the number of FMAs attributed to these layers or routines is not significant, less than $0.001 \%$ of the total number of FMAs for the 3DGAN model.

\section{RESULTS}

The 3DGAN aspires to generate scientific data leveraging an approach borrowed from the domain of visual images. It is therefore highly crucial to understand if these techniques can retain the high level of accuracy required for a scientific simulation. Each simulated image represents the pattern of energy depositions in a HEP detector, when a primary particle enters the detector volume. This pattern of energy depositions also known as "shower" has distinct physical features. These features are important information pertaining to a certain type of particle, the energy with which it strikes the detector, as well as the geometry of the detector.

The 3DGAN performance is validated by comparing GAN images to the Monte Carlo data in terms of several relevant physics distributions. Here we further compare features from images generated by networks trained using FP32 and the MP data types. The Monte Carlo data set used in this experiment represents the geometry of a sampling calorimeter. In a sampling calorimeter there are alternate arrays of passive and active layers. Energy is only recorded in the active layers and thus is a fraction of the energy of the incident particle. The sampling fraction $(S F)$ is the ratio of the energy recorded in the detector to the energy of the incoming particle
$\left(E_{p}\right)$ : it represents the detector response. Figure 6 presents the sampling fraction calculated from images simulated using Monte Carlo and 3DGAN. There is a high level of agreement for most of the $E_{p}$ range for the GAN generated images using both the FP32 and MP approaches.

The geometry of the generated shower is often used in particle identification. There can be a number of ways to quantify geometrical features. The shower shapes are the distribution of the deposited energy along a certain detector axis. The $Z$ axis is along the depth of the calorimeter, while $X$ and $Y$ axes are the transverse directions. Figure 7 compares the shower shapes for Monte Carlo images with images generated by the models trained using the FP32 and MP approaches. The top row shows the shower shapes in linear scale. The log scale plots in the bottom row are added to better appreciate the agreement at the tails of the showers. The training with MP approach is able to achieve a similar level of accuracy as the higher precision 32-bit approach.

As explained in Section [I] we created the binary analysis tool to emulate MP training, since the real hardware to perform the tests was not available at the time of the experiment. By using our developed binary analysis tool, we have been able to demonstrate that MP training can be used for HEP simulations. MP delivers the same level of accuracy as higher precision approaches implemented using FP32. Furthermore, using real hardware implementations, this approach would reduce memory storage and bandwidth requirements by nearly 50\% compared to full FP32 implementations, and increase the FMA throughput by at least twice for the same hardware vector register size. Performance improvements can be even better with dedicated wider units targeting BF16, such as the one available on the recently released Intel Cooper Lake, Nvidia Tensor Core or Google TPU.

\section{RELATED WORK}

\section{A. Machine learning in scientific applications}

The HEP community has a long tradition of using Neural Networks and Machine Learning methods (Random Forests, BDT, MLPs) to solve specific tasks, in particular related to a more efficient selection of interesting events over the overwhelming background produced at colliders such as the Large Hadron Collider (LHC). In the recent years, several studies have demonstrated the benefit of using Deep Learning (DL) to solve typical tasks related to data taking and analysis. Building on these examples, many HEP experiments are now working on integrating DL into their workflows for many different applications: from data quality assurance [38], to real-time selection of interesting collision events [39], to simulation [40] and data analysis [41]. For example, generative models, from GAN to Variational Auto-Encoders (VAE), are being tested as fast alternatives to Monte Carlo based simulation. Anomaly detection algorithms are being explored to improve data quality monitoring, to design searches for rare new-physics processes, or to analyse and prevent faults in complicated systems such as detectors and accelerators control system. 

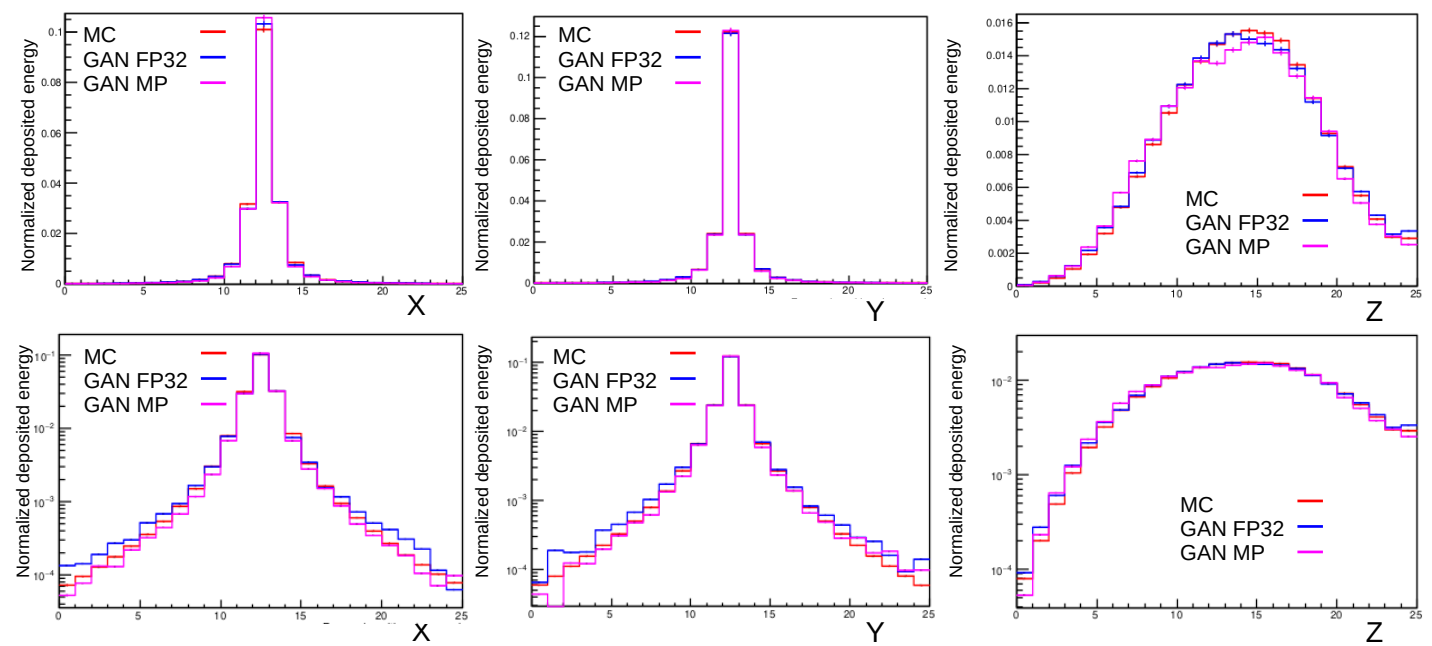

Fig. 7: Shower shapes (energy distribution) for Monte Carlo (red), GAN FP32 (blue) and GAN MP (magenta) along $X, Y$ and $Z$ axis. In linear scale(top row); and in log scale (bottom row).

Training of such models has been made tractable with the improvement of optimization methods and the advent of dedicated hardware well adapted to tackle the highly-parallelizable task of training neural networks. Memory consumption, however, still represents a limiting factor in many applications: HEP detectors output is represented by millions of read-out channels and it is usually too large to be processed as a whole. The standard approach in these cases is segmentation and selection of regions of interest for further processing. The possibility to analyse larger input samples and process deeper models represents an advantage in terms of data processing efficiency and accuracy and computing resources exploitation.

\section{B. Generative Adversarial Networks (GAN)}

HEP simulations with detailed Monte Carlo methods is a highly resource intensive task, consuming more than $50 \%$ of the Worldwide LHC grid (WLCG) resources [42]. In the past few years, development and optimisation of generative models to speedup or entirely replace Monte Carlo simulation has become a lively research field. CALOGAN [12] simulated particle showers in a simplified calorimeter conditioned on the primary particle energy $(1-100 \mathrm{GeV})$. DL was used for fast simulation of the ATLAS calorimeter [13]. Showers with energies $1-260 \mathrm{GeV}$ and pseudorapidity $|\eta|$ in range of $0.2-0.25$ were generated using both VAE and GAN. The showers were generated as a flattened array of pixels. The GAN generated showers were reported to have better agreement to the Monte Carlo data as compared to the VAE generated showers. DijetGAN [43] used GAN for simulation of diject events: a background process for important physics studies at LHC. WGAN was used to simulate the LHC detector output collapsed into a two dimensional array of cells [14]. A pretrained regressor network was incorporated into the GAN setup. The results were reported to be promising although more work was required for practical implementation.
3DGAN [15] is one of the most realistic and accurate model for the simulation of a HEP detector. The showers are generated for particles coming within the $2-500 \mathrm{GeV}$ energy range, with a speedup of several orders of magnitude. Due to the generation of 3D images, large training data and a deeper model, the training can benefit greatly from reducing the data resolution. On the other hand scientific simulation is also a performance critical task, and must retain a high level of accuracy.

\section{Mixed Precision Training}

Exploring lower precision numerical formats for DNN training has been an active research topic in the recent years. Some works propose non-standard data formats as an alternative to FP32. For example, prior work indicates that dynamic fixed-point is effective to train DNN with low-precision multipliers [17]. This approach obtains state-of-the-art results by uniformly applying the dynamic fixed-point format with different scaling factors, which are driven by the overflow rate displayed by the fixed-point numbers. Gupta et al. [26] show the benefits of applying stochastic rounding to 16-bit fixed-point multiply and add operators. This work relies on FPGA emulation to show the benefits of stochastic rounding when applied to a custom fully connected neural network dealing with the MNIST dataset. Wang et al. [19] propose to train DNNs using 8-bit floating-point (FP8) numbers, by relying on a combination of 8-bit and 16-bit arithmetic and by using stochastic rounding to obtain state-of-the-art results. Additionally, Sun et al. [44] present a novel approach similar to the previous one, but without the use of stochastic rounding; however, they require a quantization, and two newly defined FP8 numerical data types.

Recently released and announced hardware products support new numerical data types to train DNNs. For instance, Nvidia GPUs support Mixed Precision (MP) training by leveraging their tensor cores, which combine FP16 and FP32 in their 
Volta architecture [20], and more recently proposed the TF32 format using up to 19-bits [27]. Similarly, Kalamkar et al. [21] propose a MP scheme using BF16 and FP32 formats. They emulate this approach using library calls that need to be integrated into the target DNN models. The conversion from BF16 to single precision does not require a scaling factor as both types are covering the same range. Conversion from 32 to 16 bits just requires a RNE rounding operation.

FMA instructions that implement a MP approach bring significant benefits since they require less memory bandwidth and register storage than FP32 FMAs. Additionally when using $\mathrm{BF} 16$, there is essentially no significant modifications or new hyper parameters for the targeted DNN model, as it operates on the same value ranges as FP32, allowing trivial adoption of this approach by the community.

\section{CONCLUSIONS}

The use of DNN to lower the computational requirements of Monte Carlo based HEP simulations has received a lot of attention in recent years. In particular, GAN models stand out as the most accurate solutions to simulate HEP detectors. However, as models in the HEP field become more complex, requirements for memory bandwidth and capacity increase. Moreover, in a production-ready GAN algorithm capable of generating data for full scale detectors these memory-related challenges would be exacerbated.

The use of lower precision numerical formats can help alleviate these constrains. However, HEP simulations require a high level of accuracy in order to minimize uncertainty on the final physics measurements. In this paper, we undertake a study to determine if reduced precision training based on MP attains similar levels of accuracy as the default FP32 reference training.

To accomplish this, we propose a binary analysis tool that enables the emulation of lower precision numerical formats without the need for hardware support. Using this tool we show that FMA instructions are responsible for a significant chunk of the total computational workload when training well known DNN models, as well as our 3DGAN use case, for which FMAs account for $48.80 \%$ of the total of instruction count. By training the 3DGAN network for 60 epochs using a representative dataset, we have been able to show that MP training employing the BF16 numerical format is able to deliver the same level of accuracy as higher-precision approaches implemented using FP32.

\section{ACKNOWLEDGMENTS}

Marc Casas has been partially supported by the Spanish Ministry of Economy, Industry and Competitiveness under Ramon y Cajal fellowship number RYC-2017-23269. Adrià Armejach has been partially supported by the Spanish Ministry of Economy, Industry and Competitiveness under Juan de la Cierva postdoctoral fellowship number IJCI-2017-33945. John Osorio has been partially supported by the Spanish Government by FPI pre-doctoral scholarship number PRE2019090406 under project SEV-2015-0493-19-4. This work has been partially supported by Intel under the BSC-Intel collaboration.

\section{REFERENCES}

[1] Y. Lecun, L. Bottou, Y. Bengio, and P. Haffner, "Gradient-based learning applied to document recognition," in Proceedings of the IEEE, 1998, pp. 2278-2324.

[2] J. Deng, W. Dong, R. Socher, L.-J. Li, K. Li, and L. Fei-Fei, "ImageNet: A Large-Scale Hierarchical Image Database," in CVPR, 2009.

[3] A. Krizhevsky, I. Sutskever, and G. E. Hinton, "Imagenet classification with deep convolutional neural networks," in NeurIPS, 2012.

[4] C. Szegedy, W. Liu, Y. Jia, P. Sermanet, S. Reed, D. Anguelov, D. Erhan, V. Vanhoucke, and R. A., "Going deeper with convolutions," in CVPR, 2015.

[5] K. He, X. Zhang, S. Ren, and J. Sun, "Deep Residual Learning for Image Recognition," CoRR, 2015.

[6] Y. LeCun, Y. Bengio, and G. Hinton, "Deep learning," Nature, vol. 521, no. 7553, pp. 436-444, 2015. [Online]. Available: https://doi.org/10.1038/nature14539

[7] D. T. Jones, "Setting the standards for machine learning in biology," Nature Reviews Molecular Cell Biology, vol. 20, no. 11, pp. 659-660, 2019. [Online]. Available: https://doi.org/10.1038/s41580-019-0176-5

[8] S. Athey, The Impact of Machine Learning on Economics. University of Chicago Press, January 2018, pp. 507-547. [Online]. Available: http://www.nber.org/chapters/c14009

[9] A. C. Mater and M. L. Coote, "Deep learning in chemistry," Journal of Chemical Information and Modeling, vol. 59, no. 6, pp. 2545-2559, 06 2019. [Online]. Available: https://doi.org/10.1021/acs.jcim.9b00266

[10] W. Jiang, J. C. G. Higuera, B. Angles, W. Sun, M. Javan, and K. M. Yi, "Optimizing through learned errors for accurate sports field registration," in The IEEE Winter Conference on Applications of Computer Vision (WACV), March 2020.

[11] I. Goodfellow, J. Pouget-Abadie, M. Mirza, B. Xu, D. Warde-Farley, S. Ozair, A. Courville, and Y. Bengio, "Generative adversarial nets," in Advances in Neural Information Processing Systems 27, Z. Ghahramani, M. Welling, C. Cortes, N. D. Lawrence, and K. Q. Weinberger, Eds. Curran Associates, Inc., 2014, pp. 2672-2680. [Online]. Available: http://papers.nips.cc/paper/5423-generative-adversarial-nets.pdf

[12] M. Paganini, L. de Oliveira, and B. Nachman, "Calogan: Simulating 3d high energy particle showers in multilayer electromagnetic calorimeters with generative adversarial networks," Phys. Rev. D, vol. 97, p. 014021, Jan 2018. [Online]. Available: https://link.aps.org/doi/10.1103/ PhysRevD.97.014021

[13] D. Salamani et al., "Deep Generative Models for Fast Shower Simulation in ATLAS," in Proceedings, 14th International Conference on e-Science: Amsterdam, Netherlands, October 29-November 1, 2018, 2018, p. 348.

[14] V. Chekalina, E. Orlova, F. Ratnikov, D. Ulyanov, A. Ustyuzhanin, and E. Zakharov, "Generative Models for Fast Calorimeter Simulation: the LHCb case," EPJ Web Conf., vol. 214, p. 02034, 2019.

[15] G. Khattak, S. Vallecorsa, and F. Carminati, "Three dimensional energy parametrized generative adversarial networks for electromagnetic shower simulation," in 2018 25th IEEE International Conference on Image Processing (ICIP), Oct 2018, pp. 3913-3917.

[16] G. R. Khattak, S. Vallecorsa, F. Carminati, and G. M. Khan, "Particle detector simulation using generative adversarial networks with domain related constraints," in 2019 18th IEEE International Conference On Machine Learning And Applications (ICMLA), 2019, pp. 28-33.

[17] M. Courbariaux, Y. Bengio, and J. David, "Training deep neural networks with low precision multiplications," ICLR, 2015.

[18] S. Gupta, A. Agrawal, K. Gopalakrishnan, and P. Narayanan, "Deep learning with limited numerical precision," CoRR, 2015.

[19] N. Wang, J. Choi, D. Brand, C. Y. Chen, and K. Gopalakrishnan, "Training deep neural networks with 8-bit floating point numbers," NeurIPS, 2018.

[20] P. Micikevicius, S. Narang, J. Alben, G. Diamos, E. Elsen, D. Garcia, B. Ginsburg, M. Houston, O. Kuchaiev, G. Venkatesh, and H. Wu, "Mixed Precision Training," ICLR, 2018.

[21] D. Kalamkar, D. Mudigere, N. Mellempudi, D. Das, K. Banerjee, S. Avancha, D. T. Vooturi, N. Jammalamadaka, J. Huang, H. Yuen, J. Yang, J. Park, A. Heinecke, E. Georganas, S. Srinivasan, A. Kundu, M. Smelyanskiy, B. Kaul, and P. Dubey, "A Study of BFLOAT16 for Deep Learning Training," 2019. 
[22] C. K. Luk, R. Cohn, R. Muth, H. Patil, A. Klauser, G. Lowney, S. Wallace, V. J. Reddi, and K. Hazelwood, "Pin: Building customized program analysis tools with dynamic instrumentation," ACM SIGPLAN Notices, 2005.

[23] A. Paszke, S. Gross, F. Massa, A. Lerer, J. Bradbury, G. Chanan, T. Killeen, Z. Lin, N. Gimelshein, L. Antiga, A. Desmaison, A. Kopf, E. Yang, Z. DeVito, M. Raison, A. Tejani, S. Chilamkurthy, B. Steiner, L. Fang, J. Bai, and S. Chintala, "Pytorch: An imperative style, high-performance deep learning library," in Advances in Neural Information Processing Systems 32, H. Wallach, H. Larochelle, A. Beygelzimer, F. d'Alché-Buc, E. Fox, and R. Garnett, Eds. Curran Associates, Inc., 2019, pp. 8024-8035. [Online]. Available: http://papers.neurips.cc/paper/ 9015-pytorch-an-imperative-style-high-performance-deep-learning-library. pdf

[24] Y. Jia, E. Shelhamer, J. Donahue, S. Karayev, J. Long, R. Girshick, S. Guadarrama, and T. Darrell, "Caffe: Convolutional architecture for fast feature embedding," 2014.

[25] M. Abadi, A. Agarwal, P. Barham, E. Brevdo, Z. Chen, C. Citro, G. S Corrado, A. Davis, J. Dean, M. Devin, S. Ghemawat, I. Goodfellow, A. Harp, G. Irving, M. Isard, Y. Jia, R. Jozefowicz, L. Kaiser, M. Kudlur, J. Levenberg, D. Mané, R. Monga, S. Moore, D. Murray, C. Olah, M. Schuster, J. Shlens, B. Steiner, I. Sutskever, K. Talwar, P. Tucker, V. Vanhoucke, V. Vasudevan, F. Viégas, O. Vinyals, P. Warden, M. Wattenberg, M. Wicke, Y. Yu, and X. Zheng, "TensorFlow: Large-scale machine learning on heterogeneous systems," 2015, software available from tensorflow.org. [Online]. Available: http://tensorflow.org/

[26] S. Wang and P. Kanwar, "Bfloat16: The secret to high performance on cloud tpus," 2019. [Online]. Available: https://bit.ly/2SISYqq

[27] Nvidia, "Nvidia ampere whitepaper," May 2020. [Online]. Available: https://bit.ly/3dllHnV

[28] Intel, "Intel announces unmatched ai and analytics platform with new processor, memory, storage and fpga solutions," Jun 2020. [Online]. Available: https://intel.ly/3dnaWRS

[29] N. Stephens, "Bfloat16 processing for neural networks on armv8-a," August 2019. [Online]. Available: https://bit.ly/3dnzPwP

[30] A. Odena, C. Olah, and J. Shlens, "Conditional image synthesis with auxiliary classifier GANs," in Proceedings of the 34th International Conference on Machine Learning, ser. Proceedings of Machine Learning Research, D. Precup and Y. W. Teh, Eds., vol. 70. International Convention Centre, Sydney, Australia: PMLR, 06-11 Aug 2017, pp. 2642-2651. [Online]. Available: http://proceedings.mlr.press/v70/ odena17a.html

[31] R. Acciarri, C. Adams, J. Asaadi, B. Baller, T. Bolton, C. Bromberg, F. Cavanna, E. Church, D. Edmunds, A. Ereditato et al., "First observation of low energy electron neutrinos in a liquid argon time projection chamber,' Physical Review D, vol. 95, no. 7, p. 072005, 2017.

[32] Y. Chatelain, E. Petit, P. de Oliveira Castro, G. Lartigue, and D. Defour, "Automatic exploration of reduced floating-point representations in iterative methods," in Euro-Par Parallel Processing - International Conference, 2019.

[33] F. Chollet et al., "Keras," https://github.com/fchollet/keras 2015.

[34] Intel. Intel deep neural network library. [Online]. Available: https: //github.com/intel/mkl-dnn

[35] _. Intel math kernel library. [Online]. Available: https://software. intel.com/en-us/mkl

[36] D. Bahdanau, K. H. Cho, and Y. Bengio, "Neural machine translation by jointly learning to align and translate," ICLR, 2015.

[37] B. Trevett. (2020, jan) Neural machine translation by jointly learning to align and translate. [Online]. Available: https://bit.ly/381D6v4

[38] V. Azzolini, D. Bugelskis, T. Hreus, K. Maeshima, M. J. Fernandez, A. Norkus, P. J. Fraser, M. Rovere, M. A. Schneider et al., "The data quality monitoring software for the $\mathrm{cms}$ experiment at the lhc: past, present and future," in EPJ Web of Conferences, vol. 214. EDP Sciences, 2019, p. 02003.

[39] S. Giagu et al., "Fast and resource-efficient deep neural network on fpga for the phase-ii level-0 muon barrel trigger of the atlas experiment," ATL-COM-DAQ-2020-001, Tech. Rep., 2020.

[40] T. Golling, M. Duehrssen, J. Raine, G. Stewart, and D. Salamani, "Fast calorimeter simulation in atlas with dnns," ATL-COM-SOFT-2020-002, Tech. Rep., 2020.
[41] J. Ren, L. Wu, and J. M. Yang, "Unveiling cp property of top-higgs coupling with graph neural networks at the lhc," Physics Letters B, vol. 802, p. $135198,2020$.

[42] "Worldwide lhc computing grid," http://wlcg.web.cern.ch/ accessed: 2019-03-27.

[43] R. Di Sipio, M. F. Giannelli, S. K. Haghighat, and S. Palazzo, "DijetGAN: A Generative-Adversarial Network Approach for the Simulation of QCD Dijet Events at the LHC," JHEP, vol. 08, p. 110, 2020.

[44] X. Sun, J. Choi, C. Y. Chen, N. Wang, S. Venkataramani, V. Srinivasan, X. Cui, W. Zhang, and K. Gopalakrishnan, "Hybrid 8-bit Floating Point (HFP8) Training and Inference for Deep Neural Networks," in NeurIPS 2019. 